\title{
ROLE OF “ATYPICAL” MICROORGANISMS ON THE FORMATION OF BRONCHIAL ASTHMA IN CHILDREN WITH ACUTE AND RECURRENT OBSTRUCTIVE BRONCHITIS
}

\author{
OLGA V ZHUKOVA ${ }^{1 *}$, TATJANA M KONYSHKINA ${ }^{2}$, SVETLANA V KONONOVA ${ }^{1}$
}

${ }^{1}$ Department of Management and Economics of Pharmacy and Pharmaceutical Technology, Nizhny Novgorod State Medical Academy, Russia. ${ }^{2}$ Department of General and Clinical Pharmacology, Nizhny Novgorod State Medical Academy, Russia.

Email: ov-zhukova@mail.ru

Received: 16 December 2016, Revised and Accepted: 04 January 2017

ABSTRACT

Objective: This study was undertaken to determine the link between the presence of "atypical" infections in patients with acute obstructive and recurrent obstructive bronchitis (AOB/ROB) and bronchial asthma (BA) development based on the concept of risk.

Methods: The materials for the study were the data records of patients hospitalized with AOB or ROB and whose analysis was performed to identify antibodies to "atypical" microflora (796 patients). The study period was 4 years from 2008 to 2011. In the analyzed period, immunosorbent assay for the detection of antibodies to "atypical" microflora (Chlamydophila pneumoniae, Mycoplasma pneumoniae, Mycoplasma hominis) was performed. The concept of risk identification was based on the determination of the absolute risk, attributable risk (AtR), relative risk, population attributable risk, as well as on the definition of the standard errors for each type of risk and the confidence interval.

Results and Conclusion: Methodical aspects of determining the relationship between the presence of "atypical" infections in patients with AOB or ROB and BA development were based on the concept of risk. The analysis showed a direct link between the increase of cases of BA formation against the backdrop of "atypical" infections. Therefore, the performed analysis of atypical pathogens influences on BA occurrence in patients with $\mathrm{AOB} / \mathrm{ROB}$ which indicates direct dependence increase of BA incidence on atypical infection. In experimental group, Frequency of event is $14.84 \%$ in experimental group. Frequency of event is $1.67 \%$ in control group. The risk factor increases probability of event by $13.17 \%$, the risk factor increases probability of event by $13.17 \%$. Presence of atypical infection leads to increase of BA incidence by 8.9 times. Number needed to harm (NNH) is 7.59 , i.e., in the presence of atypical infection in patients with $\mathrm{AOB} / \mathrm{ROB}$, each eighth exposed person develops $\mathrm{BA}$ in addition to background level of BA incidence.

Keywords: Acute obstructive bronchitis, Recurrent obstructive bronchitis, Bronchial asthma, The concept of risk, Risk factor, The absolute risk, Relative risk, Attributable risk, Population attributable risk, Number needed to harm.

(c) 2017 The Authors. Published by Innovare Academic Sciences Pvt Ltd. This is an open access article under the CC BY license (http://creativecommons. org/licenses/by/4. 0/) DOI: http://dx.doi.org/10.22159/ajpcr.2017.v10i4.16656

\section{INTRODUCTION}

Acute obstructive bronchitis (AOB) is a common disease, which affects $10-15 \%$ of child population. $\mathrm{AOB}$ incidence is increasing globally. More than $50 \%$ of infants may develop recurrent obstructive bronchitis (ROB) in association with acute viral respiratory infection (AVRI) [1]. If bronchitis with bronchial obstructive syndrome (BOS) occurs at least 2-3 times a year, ROB develops. Bronchial asthma (BA) is also the common cause of recurrent incidence of BOS.

Nowadays, continuous increasing incidence and the severity of BA are noted. BA is often evolved in children at an early age, hence it is a considerable problem [2]. Microbe-virus associations were proved, in which one of the infectious agents is intracellular pathogen, for example, Chlamydophila pneumoniae, Mycoplasma pneumoniae, Legionella spp. etc., play an important role in bronchial obstructive disease, may cause exacerbation and severe course of disease. In recent years, numerous researches of atypical role of respiratory pathogens in recurrent BOS are performed $[3,4]$.

In epidemiological and clinical researches, often it is necessary to evaluate the power of interaction between impact and outcome (disease, complications, death, etc.) when parameters are paired, i.e., they are alternative outcomes for research subjects. In general, risk concept considers ratio between participants, who is exposed and not exposed of any factor. Hence, risk concept evaluated the effect of smoking on the occurrence of chronic obstructive pulmonary disease exacerbations [5].
The purpose of this research is to reveal the interaction between the presence of atypical infections in patients with $\mathrm{AOB}, \mathrm{ROB}$, and $\mathrm{BA}$, according to risk conception.

\section{METHODS}

Study site

The study was conducted in a hospital in Nizhny Novgorod.

\section{Study population}

The study included stroke patients who underwent treatment.

Study design

The design of the study was of retrospective type.

\section{Period of the study}

The study was performed for 4 years (2008-2011).

\section{Inclusion criteria}

In the research, we applied medical records of patients, who were hospitalized with AOB/ROB and in whom antibody test for "atypical" microflora (796 patients) was performed.

Over a period of study, enzyme immunoassay for atypical microflora (C. pneumonia, Mycoplasma pneumoniae, and Mycoplasma hominis) antibodies was performed.

IgA antibodies were detected in 256 (32.2\%) examined patients. 


\section{Methodology of data processing}

Detection risk concept was built on absolute risk (AR) detection in exposed group (ARe) and in unexposed group (ARu) (i.e., in patients with concurrent atypical infection and without concurrent atypical infection, respectively), on attributable risk (AtR), relative risk (RR), population AtR (PAR), and also on the detection of standard error for all risk types and confidence interval (CI).

\section{RESULTS}

Analysis of risk for occurrence of $\mathrm{BA}$ in patients with $\mathrm{AOB}$ and ROB begins with the construction of table of conjugate distributions (Table 1). Rows and columns of this table are arranged in certain order. Therefore, parameters that were calculated from this table in the course of research make a sense and may be interpreted correctly. The first row assigns for exposed group, which consists of children having studied risk factor, i.e., patients with BOS and concurrent atypical infection. The second row assigns for values, characterizing of BA risk in patients with BOS, but without atypical infection. The studied risk factor was absent in this group. In summary, the first group is interesting for investigators, i.e., patients in this group were exposed to risk factor. Values in the second row have been received from unexposed group, i.e., from patients without concurrent atypical infection. In the first column, it is recorded how many times the investigated event (risk of BA occurrence) was registered in exposed group (patients with concurrent atypical infection) and in unexposed group (without concurrent atypical infection). In the second column, it is recorded how many times investigated event was absent.

In the beginning, hypothesis is formed that presence of atypical infection in patients with BOS may lead to BA. First of all, AR is calculated, i.e., proportion of ill in the whole group (in exposed and unexposed groups separately). In our case, it means computation of BA risk in group of patients with/without concurrent atypical infection. By Formula (1), we calculate the frequency of BA occurrence in exposed group (with atypical infection), i.e., $14.84 \%$ :

$\operatorname{ARe}=\frac{a}{A}$

In other words, 0.1484 or $14.84 \%$ of patients in the exposed group have a risk of BA. By Formula (2), we calculate the frequency of BA occurrence in unexposed group (without atypical infection), i.e., 1.67\%:

$$
\mathrm{ARu}=\frac{\mathrm{c}}{\mathrm{B}}
$$

As a result, we have the so-called point estimates of BA occurrence frequency in experimental and control groups. Point estimates may have statistical error, hence in the next sample, we may receive different values because we calculate these frequencies not on the base of whole population but on the base of representative parts, which just approximately reflects the features of population. Therefore, it is necessary to calculate the standard error of received AR, i.e., statistical error of each frequency, which indicates the accuracy of estimate. Standard error for AR in the exposed group was calculated by Formula (3) - 0.022:

$\mathrm{S}_{\mathrm{AR}}=\sqrt{\frac{\mathrm{AR} \times(1-\mathrm{AR})}{\mathrm{n}}}$

Table 1: Table of conjugate distributions

\begin{tabular}{llll}
\hline \multirow{2}{*}{ Atypical infection } & \multicolumn{2}{l}{ Risk of BA } & \multirow{2}{*}{ Total } \\
\cline { 2 - 3 } & Yes & No & \\
\hline Present & $38(\mathrm{a})$ & $218(\mathrm{~b})$ & $256(\mathrm{~A})$ \\
Not present & $9(\mathrm{c})$ & $531(\mathrm{~d})$ & $540(\mathrm{~B})$ \\
Total & 47 (C) & $749(\mathrm{D})$ & $796(\mathrm{Q})$ \\
\hline BA. Bronchial & & &
\end{tabular}

Where $\mathrm{n}$ - volume of exposed or unexposed group, i.e., A or B.

Standard error for AR in the unexposed group was calculated similarly at 0.005 .

The received frequencies may change in case of calculation in other samples. Therefore we need to define how these changes will be significant, and minimal intervals of values involve actual precise values of required frequencies. We define minimal interval, which consists from actual value of required frequency with probability of $95 \%$. In statistics, such interval is named $95 \%$ (95\% CI). From a practical point of view, $95 \%$ CI means that $95 \%$ of all potential samples give frequencies that are included in received intervals, but $5 \%$ will be out of these intervals. In research, $95 \% \mathrm{CI}$ or $99 \% \mathrm{CI}$ is used mostly.

We calculate $95 \%$ CI for AR of exposed group at $0.1484+0.0431$ or $14.84 \pm 4.31 \%$ by Formula (4):

$\mathrm{CI}_{\mathrm{AR}}=\mathrm{AR} \pm \mathrm{t} \times \mathrm{S}$

Where $\mathrm{t}$ - critical value for statistical significance. For $95 \% \mathrm{CI}, \mathrm{t}=1.96$,

\section{S - Standard error of AR.}

In other words, minimal value of $95 \% \mathrm{CI}$ is $10.53 \%$, maximal value is $19.15 \%$, mean ARe is $14.84 \%$, and standard error is $2.20 \%$.

To summarize, the presence of concurrent atypical infections in the studied group led to BA occurrence in $14.84 \pm 2.20 \%, 95 \%$ of all possible values of incidence are included in interval at 10.53-19.15\%.

Analogically on Formula (4), 95\% CI for AR in unexposed group was calculated: $0.0167 \pm 0.0098$ or $1.67 \pm 0.98 \%$.

In other words, without concurrent atypical infection, the risk of BA occurrence in control group is $1.67 \pm 0.98 \%$. $95 \%$ of all possible values of incidence are included in interval $0.69-2.65 \%$.

Thus, absolute values of morbidity in exposed and unexposed groups were received. Therefore, under the impact, BA occurrence increases. But how significant is the role of risk factor in this increasing? AtR is estimated, which characterizes risk parts that are associated with this risk factor. AtR is calculated by Formula (5) and it is 0.317 or $13.17 \%$.

$\mathrm{AtR}=\mathrm{ARe}-\mathrm{ARu}=\frac{\mathrm{a} / \mathrm{A}}{\mathrm{c} / \mathrm{B}}$

In other words, in experimental group, Frequency of event is $14.84 \%$ in experimental group. Frequency of event is $1.67 \%$ in control group. The risk factor increases probability of event by $13.17 \%$.

For calculation of standard error of received distinction, it is necessary to compute the combined estimate of the proportion. It is 0.059 .

$\mathrm{F}=\frac{\mathrm{C}}{\mathrm{Q}}$

Then, we determined standard error of AtR by Formula (7). It was 0.0183 or $1.83 \%$.

$S_{A t R}=\sqrt{F \times(1-F) \times\left(\frac{1}{A}+\frac{1}{B}\right)}$.

Then, we calculated $95 \%$ CI for AtR: $0.1317 \pm 0.0359$ or $13.17 \% \pm 3.59 \%$.

To summarize, the presence of concurrent atypical infections in patients with $\mathrm{AOB}$ of ROB leads to increased risk of BA occurrence by $13.17 \pm 1.83 \%$. $95 \%$ of possible (true) values of difference of morbidity were included in interval $9.58-16.76 \%$. Therefore, possible (true) values, which are included in $95 \% \mathrm{CI}$, may indicate about $\mathrm{ARe}>\mathrm{AR}$, i.e., atypical infection increases of $\mathrm{BA}$ risk. 
If $\mathrm{ARe}=\mathrm{ARu}$, impact of risk factor does not change the probability of event. If $\mathrm{ARe}<\mathrm{ARu}$, impact of risk factor does not change the possibility of event.

Risk estimation methodology is based on statistical parameters (mean, mean error, $\mathrm{CI}$ ), which are based on probability theory. Therefore, when we calculate any risk, we should talk not about absolute (precision) dependence of outcome from factor but about the probability degree of this dependency. In turn, dependence of the outcome from the factor may be significant. In this case, statistical reliable probability of such dependence may be revealed in small samples. If dependence of the outcome from the factor is insignificant, for detection of statistical reliable probability, big samples are needed, sometimes whole population (e.g., population of certain region).

Analogically considering the features of CI AtR, it is necessary to analyze CI for all calculating risks. For example, in ARe and ARn, calculated earlier CI (10.53 and 19.15\%, 0.69 and 2.65\%, respectively) did not involve 0 or negative values. Consequently, these CIs may be considered statistically significant.

Through AtR, it has been demonstrated that presence of atypical infection leads to increase of BA risk on an average of $13.17 \%$.

Through calculation of RR (risk ratio or relative risk), we may demonstrate the power of dependence between impacting risk factor and outcome, i.e., how many times BA morbidity increases in the presence of atypical infection in patients with $\mathrm{AOB}$ and ROB. If $\mathrm{BA}$ occurred more often in exposed group, ratio of ARe/ARn is $>1$. If BA morbidity is equal in both groups, ARe/ARn ratio equals to 1 . Thus, according to hypothesis, from mathematical point of view, it is necessary to prove that ratio of BA occurrence in both groups is more than 1 .

RR was calculated by Formula (8) and equals to 8.9.

$\mathrm{RR}=\frac{\mathrm{ARe}}{\mathrm{ARu}}=\frac{\mathrm{a} / \mathrm{A}}{\mathrm{c} / \mathrm{B}}$

In other words, presence of atypical infection leads to increase of BA occurrence by 8.9 times. However, when we are talking about calculation, made on sample, it is necessary to show a statistical significance of received result. For this purpose, standard error of RR was calculated, it equals to 0.3619 .

$\mathrm{S}_{\mathrm{OP}}=\sqrt{\frac{1-\mathrm{AP}_{\ni}}{\mathrm{a}}+\frac{1-\mathrm{APH}}{\mathrm{c}}}$

By Formula (10), we calculated 95\% CI of RR, received value of $8.9 \pm 4.71 \%$.

$\mathrm{CI}_{\mathrm{RR}}=\mathrm{RR} \pm \operatorname{Exp}\left(\ln \frac{\mathrm{ARe}}{\mathrm{ARu}} \times \mathrm{t} \times \mathrm{S}\right)$

The received values of RR are $8.9 \pm 0.3619$ with $95 \%$ CI of $4.19-13.61$ (Fig. 1). Analogically to AtR possible (true) values, involving in $95 \% \mathrm{CI}$, may indicate that:

- $\quad \mathrm{RR}>1$, i.e., atypical microflora influence increases the risk of $\mathrm{BA}$;

- $\mathrm{RR}=1$, i.e., atypical microflora influence does not change the risk of $\mathrm{BA}$;

- $\mathrm{RR}<1$, i.e., atypical microflora influence decreases the risk of BA.

Therefore, 95\% CI values indicate the importance of our hypothesis about atypical microflora influence on BA occurrence.

PAR is absolute difference of values (or risk) in whole population and in the unexposed group. PAR similar to AtR. But population component describes the risk component in the whole population. PAR depends upon how widespread risk factors in this population. PAR varies depending on risk factor distribution in population.
By Formula (11), PAR value was calculated, it received 0.042 or $4.2 \%$.

$$
\mathrm{PAR}=\frac{\mathrm{C}}{\mathrm{Q}}-\frac{\mathrm{c}}{\mathrm{B}}
$$

In other words, the presence of atypical infection leads to morbidity increase in the whole population by $4.2 \%$.

For calculation of standard error of received difference, it is necessary to compute the combined estimate of the proportion. Standard error of AR in the exposed group was calculated by Formula (6) - 0.059 .

By Formula (12), we can calculate mean error, it received 0.0134 or $1.34 \%$.

$\mathrm{S}_{\mathrm{PAR}}=\sqrt{F \times(1-\mathrm{F}) \times\left(\frac{1}{\mathrm{Q}}+\frac{1}{\mathrm{~B}}\right)}$

By Formula (13), we can calculate $95 \%$ CI PAR. It is $0.042 \pm 0.026$ or $4.20 \pm 2.60 \%$

$\mathrm{CI}_{\mathrm{PAR}}=\mathrm{PAR} \pm \mathrm{t} \times \mathrm{S}$

Thus, the presence of atypical infection increases BA morbidity in population by $4.20 \pm 1.34 \%, 95 \%$ CI equals from $1.6 \%$ to $6.8 \%$ (Fig. 2).

There is another parameter, which is a derivative from AtR, number needed to harm (NNH) or number needed to treat (NNT). If exposure factor presumably caused negative changes in health status, we are speaking about NNH. If exposure factor improves health status (e.g., new treatment method decreases the number of recurrences, prophylactic measures decrease of incidence), we mean NNT. Both parameters are ratio 1 to AtR (14).

$\mathrm{NNH}(\mathrm{NNT})=\frac{1}{\mathrm{AtR}}$

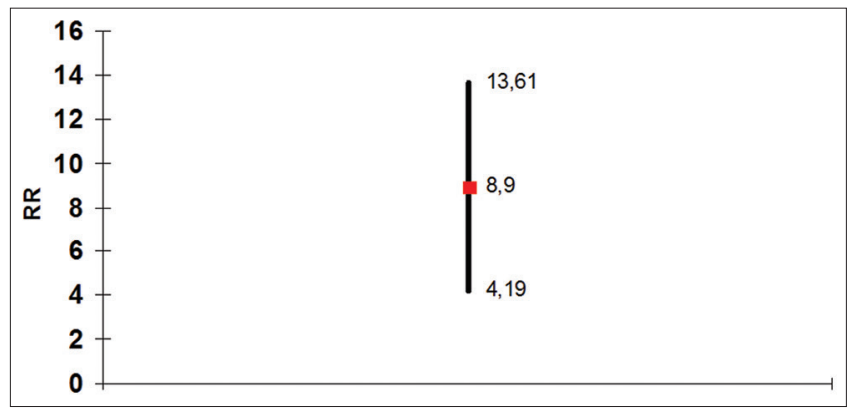

Fig. 1: Corridor fluctuation relative risk values with $95 \%$ confidence interval of bronchial asthma by persistent infection with atypical pathogens

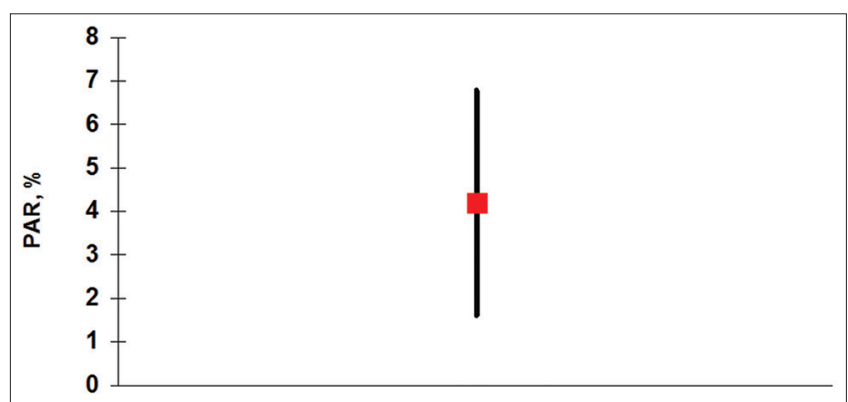

Fig. 2: Corridor fluctuation population attributable risk values with $95 \%$ confidence interval of bronchial asthma by persistent infection with atypical pathogens 
$\mathrm{NNH}$ for atypical infection in the above-mentioned example is 7.59.

In other words, in the presence of atypical infection in patients with $\mathrm{AOB} / \mathrm{ROB}$, each eighth exposed person develops BA in addition to the background level of BA incidence.

\section{DISCUSSION}

The results on the effect of atypical microorganisms on the formation of BA are consistent with previous results. Etiology of AOB and its recurrences can be different: It can be viral, bacterial, fungal, parasitical, mixed, or allergic. From a practical perspective, in pediatrics, there are viral and bacterial bronchitis. In some cases, bronchitis may be caused by atypical microflora (Mycoplasma, Chlamydophila, Legionella, Pneumocystis), sometimes by mycology. Influenza and Para influenza viruses, adenoviruses, respiratory syncytial virus, coronavirus, rhinovirus, Coxsackie, and ECHO viruses are the most frequently occurring among viral causative agents. Pneumococcus (Streptococcus pneumoniae), Haemophilus influenzae, and Moraxella (Moraxella catarrhalis) are leading among bacterial causative agents at the present time [6].

Mycoplasmal and chlamydial etiologies of bronchitis occur most frequently in children at their first year of life and after 10 years, it can amount to $25-40 \%$ of cases [7].

It is important in each case to determine the cause of recurring and make a true diagnosis. In many cases, BA is a cause. For a long time disguising under the mask of acute respiratory infection with AOB, BA remains undiagnosed timely and, therefore, patients do not receive treatment. Only after years, it is founded in part of children that the so-called obstructive bronchitis turns into typical BA. According to the results of long-term follow-up (Yu and Mizernitsky, 2005), 4-8 years after hospitalization associated with expressed BO with acute respiratory infection, more than half of the examined children suffered from typical BA that had not been diagnosed in the early age. Diagnostics and timely beginning of corresponding therapy in a large part determine disease prediction.

According to multiple data from literature, the most important aspect for patients with BO of relapsing course (including those with BA) concerns studying the role of microbial and viral associations when one of the agents is the intracellular causative agent ( $C$. pneumonia, Mycoplasma pneumoniae, Legionella spp., and others) $[8,9]$. It has been established that intracellular causative agents can both cause debut of bronchial obstruction disease and trigger its aggravation and perform severe variants of treatment $[10,11]$.

It is known that Chlamydophila and Mycoplasma can actively affect immune response of a child contributing, from one point, to secondary introduction of infection and, from the other, to increase bronchial hyperactivity and development of bronchospasm. Thus, frequency of
BO development accompanied by respiratory infection of chlamydial etiology amounts to $18-58 \%$ [12]. Studies of antibacterial therapy of AOB, which conducted by us previously, show high efficiency macrolide in comparison with beta-lactams. This fact confirms the high frequency of proliferation of atypical microorganisms in AOB in children [13].

\section{CONCLUSION}

Therefore, the performed analysis of atypical pathogens influences on BA occurrence in patients with $\mathrm{AOB} / \mathrm{ROB}$ which indicates direct dependence increase of BA incidence on atypical infection. In experimental group, Frequency of event is $14.84 \%$ in experimental group. Frequency of event is $1.67 \%$ in control group. The risk factor increases probability of event by $13.17 \%$. The presence of atypical infection leads to increase of BA incidence by 8.9 times. NNH is 7.59, i.e., in the presence of atypical infection in patients with $\mathrm{AOB} / \mathrm{ROB}$, each eighth exposed person develops BA in addition to background level of BA incidence.

\section{REFERENCES}

1. Mizernitskiy YL, Sorokina EV. Modern approaches to the treatment of acute bronchitis in children. Trudnyy Patsient 2008;9:4-8.

2. Geppe NA. Topicality of bronchial asthma in children. Pediatriya 2012;3:76-82.

3. Sereda EV, Lukina OF, Selimzyanova LR. Mechanisms of bronchial obstruction and therapeutic tactics for bronchitis in children. Pediatriya 2010;5:77-86

4. Bacharier LB, Boner A, Carlsen KH, Eigenmann PA, Frischer T, Götz M, et al. Diagnosis and treatment of asthma in childhood: A PRACTALL consensus report. Allergy 2008;63(1):5-34

5. Zhukova OV, Konyshkina TM, Kononova SV. The concept of risk factors in assessing the impact of smoking on an exacerbation of chronic obstructive pulmonary disease. Ter Arkh 2015;87(3):23-6.

6. Dzh B. Infections of Respiratory Tract. Moscow, Saint Petersburg: BINOM, Nevskiy dialekt. 2000. p. 192.

7. Samsygina GA. Antibiotics in the treatment of acute bronchitis in children. Lech Vrach 2001;1:12-5.

8. Cook PJ, Honeybourne D. Clinical aspects of Chlamydia pneumoniae infection. Presse Med 1995;24:278-82.

9. Woodcock A, Lowe LA, Murray CS, Simpson BM, Pipis SD, Kissen $\mathrm{P}$, et al. Early life environmental control: Effect on symptoms, sensitization, and lung function at age 3 years. Am J Respir Crit Care Med 2004;170:433-9.

10. Dietzsch HJ, Rupprecht E, Wunderlich P. Epidemiologic and bronchologic aspects of chronic bronchitis in childhood (author's transl). Padiatr Padol 1975;10(2):176-83.

11. Hahn DL. Antichlamydial antimicrobial therapy for asthma. Arch Pediatr Adolesc Med 1995;149(2):219-21.

12. Zaytseva OV. Problems of diagnosis and treatment of respiratory infections caused by "atypical" pathogens. Trudnyy Patsient 2010;9:4-8.

13. Zhukova OV, Konyshkina TM, Kononova SV. Fishburne's method and the classical method of pharmacoeconomic analysis in the evaluation of antibiotic treatment of acute and recurrent bronchitis in children. Int $\mathrm{J}$ Pharm Pharm Sci 2015;7(11):185-90. 